MATHEMATICS OF COMPUTATION

Volume 67, Number 221, January 1998, Pages 209-220

S $0025-5718(98) 00893-X$

\title{
CONVERGENCE OF NON-STATIONARY PARALLEL MULTISPLITTING METHODS FOR HERMITIAN POSITIVE DEFINITE MATRICES
}

\author{
M. JESÚS CASTEL, VIOLETA MIGALLÓN, AND JOSÉ PENADÉS
}

\begin{abstract}
Non-stationary multisplitting algorithms for the solution of linear systems are studied. Convergence of these algorithms is analyzed when the coefficient matrix of the linear system is hermitian positive definite. Asynchronous versions of these algorithms are considered and their convergence investigated.
\end{abstract}

\section{INTRODUCTION}

In this paper, we study the parallel solution of linear systems of the form

$$
A x=b,
$$

where $A \in \mathbb{C}^{n \times n}$ is a nonsingular matrix and $x, b \in \mathbb{C}^{n}$.

In order to get an iterative method to solve system (1.1) on a parallel computer, O'Leary and White [23] introduced the multisplitting technique. Later, this technique was studied by many authors; see e.g., Frommer and Mayer [11], [12], Neumann and Plemmons [22], or White [28], [29], [30].

The multisplitting method (see [23]) consists of having a collection of splittings

$$
A=F_{j}-G_{j}, \quad 1 \leq j \leq r, \quad \operatorname{det}\left(F_{j}\right) \neq 0,
$$

and diagonal nonnegative weighting matrices $E_{j}, 1 \leq j \leq r$, which add to identity, and the following algorithm is performed.

Algorithm 1. (Multisplitting).

Given the initial vector $x^{(0)}$.

For $l=0,1,2, \ldots$, until convergence.

$$
\begin{aligned}
& \text { For } j=1 \text { to } r \\
& \qquad F_{j} y_{j}=G_{j} x^{(l)}+b \\
& x^{(l+1)}=\sum_{j=1}^{r} E_{j} y_{j} .
\end{aligned}
$$

Received by the editor February 2, 1996 and, in revised form, July 29, 1996.

1991 Mathematics Subject Classification. Primary 65F10, 65F15.

Key words and phrases. Non-stationary methods, asynchronous iterations, linear systems, multisplitting, hermitian matrix, positive definite matrix.

This research was supported by Spanish CICYT grant number TIC96-0718-C02-02.

(C)1998 American Mathematical Society 
As it can be appreciated, Algorithm 1 corresponds to the following iteration

$$
x^{(l+1)}=\sum_{j=1}^{r} E_{j} P_{j} x^{(l)}, \quad l=0,1,2, \ldots,
$$

where the operators $P_{j}: \mathbb{C}^{n} \longrightarrow \mathbb{C}^{n}, 1 \leq j \leq r$, are defined as

$$
P_{j} x=F_{j}^{-1} G_{j} x+F_{j}^{-1} b .
$$

Thus, iteration (1.3) can be rewritten as

$$
x^{(l+1)}=T x^{(l)}+\sum_{j=1}^{r} E_{j} F_{j}^{-1} b, \quad l=0,1,2, \ldots,
$$

where $T=\sum_{j=1}^{r} E_{j} F_{j}^{-1} G_{j}$ is the iteration matrix.

Conditions on the splittings (1.2) and on the weighting matrices which ensure the convergence of Algorithm 1 in some important cases were given by O'Leary and White [23]. In particular they showed that convergence (i.e., $\rho(T)<1$, where $\rho(T)$ denotes the spectral radius of $T$ ) when $A$ is a symmetric positive definite matrix and the splittings (1.2) are $P$-regular (see definitions in Section 2). In an efficient implementation of a multisplitting method on a multiprocessor system, each operator $P_{j}$, defined in (1.4), represents the task assigned to one of the $r$ processors to obtain its local approximation. Each local approximation is updated exactly once using the same previous iterate $x^{(l)}$. However, it is possible to update that approximation more than once using different iterates computed earlier. In this case we call this method a non-stationary method. The main idea of these methods is that at the $l$ th iteration each processor $j$ solves the system defined by its operator $P_{j}, q(l, j)$ times, updating each time the right-hand side by using the new calculated vector, i.e.

Algorithm 2. (Non-Stationary Multisplitting).

Given the initial vector $x^{(0)}$

$$
\begin{aligned}
& \text { For } l=0,1,2 \ldots, \text { until convergence } \\
& \text { In processor } j, j=1 \text { to } r \\
& \qquad \begin{array}{c}
(0) \\
y_{j}
\end{array} x^{(l)} \\
& \text { For } k=1 \text { to } q(l, j) \\
& F_{j} y_{j}^{(k)}=G_{j} y_{j}^{(k-1)}+b \\
& x^{(l+1)}=\sum_{j=1}^{r} E_{j} y_{j}^{(q(l, j))} .
\end{aligned}
$$

Bru, Elsner and Neumann [3] showed the convergence of this algorithm when $A^{-1} \geq 0$ and the splittings $(1.2)$ are weak regular $\left(F_{j}^{-1} \geq 0\right.$ and $F_{j}^{-1} G_{j} \geq 0,1 \leq$ $j \leq r)$. They used the term chaotic for these non-stationary methods; however we have chosen the non-stationary term since in the classical literature chaotic is synonymous with asynchronous, e.g., as in [8]. 
In Algorithm 2 a relaxation parameter $\omega \in \mathbb{R}, \omega \neq 0$, can be introduced by replacing the computation of $x^{(l+1)}$ in (1.6) with the equation

$$
x^{(l+1)}=\omega \sum_{j=1}^{r} E_{j} y_{j}^{(q(l, j))}+(1-\omega) x^{(l)} .
$$

Clearly, with $\omega=1$, equation (1.6) is recovered. In the case of $\omega \neq 1$ we have a Relaxed Non-stationary Multisplitting Algorithm (Algorithm 3). The convergence of algorithms 2 and 3 when $A$ is an $H$-matrix was studied by Mas, Migallón, Penadés and Szyld [20]. Furthermore, in [20] the authors report computational results of those algorithms on a multiprocessor system that show a better behavior of the non-stationary models than the stationary ones (Algorithm 1). For a background on parallel non-stationary models see also [5], [6], [7], [13], [14] or [15].

In this paper we concentrate our study on the case where $A$ is a hermitian positive definite matrix. In Section 3 we study the convergence of Algorithm 2, together with its relaxed version, Algorithm 3. We also study their extension to asynchronous algorithms where the solution of the systems (1.5) proceed in each processor without waiting for the completion of the computation of the iterates in the other processors; see Section 4. Previously, in Section 2 we present some notation, definitions and preliminary results which we refer to later.

\section{NOTATION AND PRELIMINARIES}

For any matrix $A \in \mathbb{C}^{n \times n},|A|$ denotes the matrix whose entries are the modulus of the corresponding entries of $A$; the matrices $A^{T}$ and $A^{H}$ denote respectively the transpose and the conjugate transpose of $A$. Similarly the transpose and the conjugate transpose of a vector $x \in \mathbb{C}^{n}$ are denoted by $x^{T}$ and $x^{H}$, respectively. A matrix $A \in \mathbb{C}^{n \times n}$ is symmetric if $A=A^{T}$, and hermitian if $A=A^{H}$. Obviously a real symmetric matrix is a special case of a hermitian matrix.

Recall that a complex, not necessarily hermitian matrix $A$, is positive definite if the real part of $x^{H} A x$ is positive, for all complex $x \neq 0$. When $A$ is hermitian, this is equivalent to requiring that $x^{H} A x>0$, for all complex $x \neq 0$. In addition, a general matrix $A$ is positive definite if and only if the hermitian matrix $A+A^{H}$ is positive definite. Let $A \in \mathbb{C}^{n \times n}$ be a hermitian positive definite matrix, then $\langle x, y\rangle=\left(x^{H} A y\right)^{1 / 2}$ defines an inner product on $\mathbb{C}^{n}$. Hence, $\|x\|_{A}=\left(x^{H} A x\right)^{1 / 2}$ is a vector norm on $\mathbb{C}^{n}$. The matrix norm induced by that vector norm will also be denoted by $\|\cdot\|_{A}$. In addition, $\|\cdot\|_{\infty}$ denotes the infinite matrix norm. Let $A \in \mathbb{C}^{n \times n}$, the splitting $A=M-N$ is called $P$-regular if the matrix $M^{H}+N$ is positive definite; see e.g., [2], [24], [25], for an extensive bibliography on hermitian matrices and positive definite matrices.

The following theorem gives convergence conditions for iterative methods based on a single splitting $A=M-N$, when $A$ is a hermitian matrix. The proof can be found, e.g, in [2].

Theorem 2.1. Let $A=M-N$ be a P-regular splitting of a hermitian matrix $A$. Then $\rho\left(M^{-1} N\right)<1$ if and only if $A$ is positive definite.

A similar result for Algorithm 1 was obtained by O'Leary and White [23], assuming that the weighting matrices $E_{j}, 1 \leq j \leq r$, of Algorithm 1 have the form $E_{j}=\alpha_{j} I$. Although that result was given for symmetric matrices, it can be extended without difficulty to hermitian matrices. 
Theorem 2.2. Let $A=F_{j}-G_{j}, 1 \leq j \leq r$, be P-regular splittings of a hermitian positive definite matrix, and $E_{j}=\alpha_{j} I, 1 \leq j \leq r$, with $\alpha_{j}>0$ and $\sum_{j=1}^{r} \alpha_{j}=1$. Then Algorithm 1 is convergent.

We point out that if the weighting matrices $E_{j}$ are not of the form $E_{j}=\alpha_{j} I$, Algorithm 1 may not converge when $A$ is hermitian positive definite, even though the splittings of $A$ are $P$-regular. Here we report an example, different to that used in [23], that illustrates this situation. Later, we will use this example for the non-stationary methods.

Example 2.3. Consider

$$
A=\left[\begin{array}{ll}
0.75 & 0 \\
0 & 0.75
\end{array}\right]=F_{1}-G_{1}=F_{2}-G_{2},
$$

where

$$
F_{1}=\left[\begin{array}{rr}
0.3934 & -2.0660 \\
2.0660 & 7.6244
\end{array}\right], \quad G_{1}=\left[\begin{array}{rr}
-0.3566 & -2.0660 \\
2.0660 & 6.8744
\end{array}\right],
$$

and

$$
F_{2}=\left[\begin{array}{rr}
7.6244 & 2.0660 \\
-2.0660 & 0.3934
\end{array}\right], \quad G_{2}=\left[\begin{array}{rr}
6.8744 & 2.0660 \\
-2.0660 & -0.3566
\end{array}\right] .
$$

Setting $E_{1}=\left[\begin{array}{ll}0 & 0 \\ 0 & 1\end{array}\right], E_{2}=\left[\begin{array}{ll}1 & 0 \\ 0 & 0\end{array}\right]$, the iteration matrix $T=E_{1} F_{1}^{-1} G_{1}+$ $E_{2} F_{2}^{-1} G_{2}=\left[\begin{array}{ll}0.9594 & 0.2132 \\ 0.2132 & 0.9594\end{array}\right]$, has spectral radius equal to 1.1726, and so Algorithm 1 is not convergent. However, Algorithm 1 converges setting other weighting matrices that do not satisfy Theorem 2.2. For example, if the above matrices $E_{1}$ and $E_{2}$ are interchanged, the spectral radius of the resulting iteration matrix is 0.4264 .

The following lemmas will be very useful in our convergence analysis.

Lemma 2.4. Given a nonsingular matrix $A$ and a matrix $T$ such that $(I-T)^{-1}$ exists, there is a unique pair of matrices $P, Q$ such that $P$ is nonsingular, $T=P^{-1} Q$ and $A=P-Q$. The matrices are $P=A(I-T)^{-1}$ and $Q=P-A$.

Proof. See Lemma 8 of Lanzkron, Rose and Szyld [18].

Lemma 2.5. Let $T^{(l)}, l=0,1,2, \ldots$, be a sequence of square complex matrices. If there exists a matrix norm $\|\cdot\|$ such that $\left\|T^{(l)}\right\| \leq \theta<1, l=0,1,2, \ldots$, then $\lim _{l \rightarrow \infty} T^{(l)} T^{(l-1)} \cdots T^{(0)}=0$.

Proof. See Lemma 2 of Bru and Fuster [4].

Lemma 2.6. Let $A$ be a hermitian positive definite matrix. If the splittings $A=$ $F-G=P-Q$ are $P$-regular, then the matrix $T=P^{-1} Q F^{-1} G$ has spectral radius less that one. Moreover, the unique splitting $A=B-C$ induced by the iteration matrix $T$, such that $T=B^{-1} C$, is also $P$-regular.

Proof. See Theorem 4.6 of Benzi and Szyld [1]. This theorem was given for symmetric matrices. The hermitian case is analogous. 


\section{Convergence OF NON-STAtionary Methods}

Given an initial vector $x^{(0)}$, the Non-stationary Multisplitting Algorithm 2 produces the sequence of vectors

$$
x^{(l+1)}=\sum_{j=1}^{r} E_{j} P_{j}^{q(l, j)} x^{(l)}, \quad l=0,1,2, \ldots,
$$

where the operators $P_{j}, 1 \leq j \leq r$, are defined in (1.4). We rewrite (3.1) as

$$
x^{(l+1)}=T^{(l)} x^{(l)}+c^{(l)}, \quad l=0,1,2, \ldots,
$$

where $T^{(l)}$ are the iteration matrices

$$
T^{(l)}=\sum_{j=1}^{r} E_{j}\left(F_{j}^{-1} G_{j}\right)^{q(l, j)}, \quad l=0,1,2 \ldots
$$

and

$$
c^{(l)}=\sum_{j=1}^{r} E_{j}\left(\sum_{i=0}^{q(l, j)-1}\left(F_{j}^{-1} G_{j}\right)^{i}\right) F_{j}^{-1} b, \quad l=0,1,2 \ldots
$$

The exact solution of the linear system (1.1) is a fixed point of the operators $P_{j}, 1 \leq j \leq r$. Then, using error analysis, it is easy to see that the sequence of vectors generated by iteration (3.1) (or equivalently, iteration (3.2)) converges to the solution of the linear system (1.1) if and only if $\lim _{l \rightarrow \infty} T^{(l)} T^{(l-1)} \cdots T^{(0)}=0$.

The following lemma is very useful when analyzing the iteration matrices (3.3).

Lemma 3.1. Let $A$ be a hermitian positive definite matrix. Assume the splitting $A=F-G$ is $P$-regular. Given $s \geq 1$, there exists a unique splitting $A=M-N$ such that $\left(F^{-1} G\right)^{s}=M^{-1} N$. Moreover, the splitting is P-regular.

Proof. The proof of the existence and uniqueness follows from Theorem 2.1 and Lemma 2.4. To show the $P$-regularity we proceed by induction. For $s=1$ the result follows from the uniqueness. Suppose that the result is true for $s-1$, that is, we know that the unique splitting $A=P-Q$, such that $\left(F^{-1} G\right)^{s-1}=P^{-1} Q$, is $P$ regular. Now, using Lemma 2.6 with the $P$-regular splittings $A=F-G=P-Q$ the proof is completed.

Theorem 3.2. Let $A$ be a hermitian positive definite matrix. Let $A=F_{j}-G_{j}, 1 \leq$ $j \leq r$, be $P$-regular splittings and $E_{j}=\alpha_{j} I, 1 \leq j \leq r$, with $\alpha_{j}>0$ and $\sum_{j=1}^{r} \alpha_{j}=1$.

Then, there exists a unique splitting $A=R^{(l)}-S^{(l)}$, induced by each iteration matrix $T^{(l)}, l=0,1,2, \ldots$, defined in $(3.3)$, such that $T^{(l)}=\left(R^{(l)}\right)^{-1} S^{(l)}$. Moreover, that splitting is $P$-regular.

Proof. Since $A=F_{j}-G_{j}, 1 \leq j \leq r$, are $P$-regular splittings of a hermitian positive definite matrix then, from Lemma 3.1, for each $j, l$ there exists a unique $P$-regular splitting $A=M_{j}^{(l)}-N_{j}^{(l)}$ such that $\left(F_{j}^{-1} G_{j}\right)^{q(l, j)}=\left(M_{j}^{(l)}\right)^{-1} N_{j}^{(l)}$. Then, the iteration matrices $(3.3)$ can be written as

$$
T^{(l)}=\sum_{j=1}^{r} E_{j}\left(F_{j}^{-1} G_{j}\right)^{q(l, j)}=\sum_{j=1}^{r} E_{j}\left(M_{j}^{(l)}\right)^{-1} N_{j}^{(l)}, \quad l=0,1,2, \ldots
$$


Now, for each fixed $l, T^{(l)}$ is the iteration matrix corresponding to Algorithm 1 by setting the splittings $A=M_{j}^{(l)}-N_{j}^{(l)}, 1 \leq j \leq r$. Since these splittings are $P$ regular, Theorem 2.2 ensures that $\rho\left(T^{(l)}\right)<1$. On the other hand, since each $M_{j}^{(l)}$ is positive definite, the matrix $\sum_{j=1}^{r} E_{j}\left(M_{j}^{(l)}\right)^{-1}$ is also positive definite, and therefore it is nonsingular. Thus, each matrix $T^{(l)}, l=0,1,2, \ldots$, is also the iteration matrix of an iterative method based on the single splitting

$$
A=R^{(l)}-S^{(l)},
$$

where $R^{(l)}=\left(\sum_{j=1}^{r} E_{j}\left(M_{j}^{(l)}\right)^{-1}\right)^{-1}$, and $S^{(l)}=R^{(l)} T^{(l)}$. Since $\rho\left(T^{(l)}\right)<1$, from Lemma 2.4 these splittings are unique. Moreover, from Theorem 3.2 of [21], it follows that the splittings (3.4) are $P$-regular.

From the above theorem follows the convergence of Algorithm 2 when the number of times $q(l, j)$ that the $j$ th processor works is fixed in each iteration $l$. That is, if $q(l, j)=q(j), l=0,1,2, \ldots, 1 \leq j \leq r$, then there is a unique iteration matrix $T=T^{(l)}, l=0,1,2, \ldots$; thus, from Theorem 3.2, $\rho(T)<1$. However, since the product of convergent matrices may not tend to zero (see e.g., Johnson and Bru [17], or Robert, Charnay and Musy [27]), then other tools are needed to show the convergence of Algorithm 2 for any sequence of integers $q(l, j) \geq 1$. So, we have the following result.

Theorem 3.3. Let $A$ be a hermitian positive definite matrix. Let $A=F_{j}-G_{j}, 1 \leq$ $j \leq r$, be $P$-regular splittings and $E_{j}=\alpha_{j} I, 1 \leq j \leq r$, with $\alpha_{j}>0$ and $\sum_{j=1}^{r} \alpha_{j}=1$. Assume that $q(l, j) \geq 1,1 \leq j \leq r, l=0,1,2, \ldots$. Then, the Non-stationary Multisplitting Algorithm 2 converges to the solution of the linear system (1.1), for any initial vector $x^{(0)}$.

Proof. Since $A=F_{j}-G_{j}, 1 \leq j \leq r$, are $P$-regular splittings of a hermitian matrix, the matrix $A-\left(F_{j}^{-1} G_{j}\right)^{H} A\left(F_{j}^{-1} G_{j}\right)=\left(F_{j}^{-1} A\right)^{H}\left(F_{j}^{H}+G_{j}\right)\left(F_{j}^{-1} A\right)$ is hermitian positive definite. Then, letting the vector norm $\|\cdot\|_{A}$, we have

$$
\left\|F_{j}^{-1} G_{j} x\right\|_{A}^{2}=x^{H}\left(F_{j}^{-1} G_{j}\right)^{H} A\left(F_{j}^{-1} G_{j}\right) x<x^{H} A x=\|x\|_{A}^{2} \text {, for all } x \neq 0 .
$$

Hence $\left\|F_{j}^{-1} G_{j}\right\|_{A}<1,1 \leq j \leq r$, and there exists a real constant $0 \leq \theta<1$ such that $\left\|F_{j}^{-1} G_{j}\right\|_{A} \leq \theta<1,1 \leq j \leq r$. Hence, for all $l=0,1,2, \ldots$, we have

$$
\left\|T^{(l)}\right\|_{A}=\left\|\sum_{j=1}^{r} E_{j}\left(F_{j}^{-1} G_{j}\right)^{q(l, j)}\right\|_{A} \leq \sum_{j=1}^{r} \alpha_{j}\left\|\left(F_{j}^{-1} G_{j}\right)^{q(l, j)}\right\|_{A} \leq \theta<1 .
$$

From Lemma 2.5, this implies that $\lim _{l \rightarrow \infty} T^{(l)} T^{(l-1)} \cdots T^{(0)}=0$, and then the proof is completed.

Theorem 3.3 generalizes Theorem 1.b of [23] (Theorem 2.2 of Section 2) for the non-stationary case, and it gives an alternative proof of the convergence result by O'Leary and White [23]. 
Note that in Theorem 3.3 we have restricted the weighting matrices in the same way as in Theorem 2.2. If we consider the splittings of Example 2.3 and we compute the iteration matrices of Algorithm 2, setting $q(l, 1)=q(l, 2)=2$ or $3, l=0,1,2, \ldots$, we obtain $\rho\left(T^{(l)}\right)>1, l=0,1,2, \ldots$, in the first choice of weighting matrices. However, if we set $q(l, 1)=q(l, 2)=4, l=0,1,2, \ldots$, we get $\rho\left(T^{(l)}\right)<1, l=0,1,2, \ldots$, in both choices of weighting matrices. Consequently it motivates the convergence study of Algorithm 2 without the additional hypothesis $E_{j}=\alpha_{j} I, 0<\alpha_{j} \leq 1$. The following theorem shows the convergence of Algorithm 2 when each processor performs enough local iterations (1.5).

Theorem 3.4. Let $A$ be a hermitian positive definite matrix. Let $A=F_{j}-G_{j}, 1 \leq$ $j \leq r$, be $P$-regular splittings. Assume further that $\lim _{l \rightarrow \infty} q(l, j)=\infty, 1 \leq j \leq r$. Then the Non-stationary Multisplitting Algorithm 2 converges to the solution of the linear system (1.1), for any initial vector $x^{(0)}$.

Proof. Since $A=F_{j}-G_{j}, 1 \leq j \leq r$, are $P$-regular splittings of a hermitian positive definite matrix, by Theorem 2.1, $\rho\left(F_{j}^{-1} G_{j}\right)<1,1 \leq j \leq r$. Then, $\lim _{i \rightarrow \infty}\left(F_{j}^{-1} G_{j}\right)^{i}=0,1 \leq j \leq r$. Thus, given an $\epsilon>0$, there exists an integer $i_{0}$ such that for any matrix norm $\|\cdot\|,\left\|\left(F_{j}^{-1} G_{j}\right)^{i}\right\| \leq \epsilon$, for all $i \geq i_{0}$. Particularly, we can choose the infinite norm. Since $\lim _{l \rightarrow \infty} q(l, j)=\infty$, there exists an $l_{0}$ such that

$$
\left\|\left(F_{j}^{-1} G_{j}\right)^{q(l, j)}\right\|_{\infty} \leq \epsilon, \quad \forall l \geq l_{0}, \quad 1 \leq j \leq r .
$$

Hence, for $l \geq l_{0}$,

$$
\left\|T^{(l)}\right\|_{\infty}=\left\|\sum_{j=1}^{r} E_{j}\left(F_{j}^{-1} G_{j}\right)^{q(l, j)}\right\|_{\infty} \leq \max _{1 \leq j \leq r}\left\|\left(F_{j}^{-1} G_{j}\right)^{q(l, j)}\right\|_{\infty} \leq \epsilon .
$$

Setting $\epsilon<1$ the convergence of Algorithm 2 follows from Lemma 2.5.

It is well known when the Jacobi, Gauss-Seidel and SOR splittings (and their block splitting versions) of a hermitian positive definite matrix are $P$-regular; see e.g., [2] and [25]. Another class of $P$-regular splittings of a hermitian positive definite matrix $A$ is the unique splitting induced by the iteration matrix of an alternating iterative method of the form

$$
x^{(l+1 / 2)}=M^{-1} N x^{(l)}+M^{-1} b, \quad x^{(l+1)}=P^{-1} Q x^{(l+1 / 2)}+P^{-1} b, \quad l=0,1,2, \ldots,
$$

where $A=M-N=P-Q$ are $P$-regular splittings; see [1]. So, the SSOR splitting is an important example of this class. Besides SSOR, one can consider, for example, the alternating iterations based on a splitting of the form $A=A_{1}+A_{2}$, where $A_{1}, A_{2}$ are positive definite and $A_{2}=A_{1}^{T}$; choosing $M=\beta I+A_{1}, N=\beta I-A_{2}, P=$ $\beta I+A_{2}$ and $Q=\beta I-A_{1}$, with $\beta>0$, the splittings $A=M-N=P-Q$ are $P$-regular; see e.g., [19]. Furthermore, there are other ways to construct $r$ $P$-regular splittings of a hermitian positive definite matrix; for example, see [23], let $A=\sum_{j=1}^{r} A_{j}$ be a hermitian positive definite matrix, and let $D_{j}, 1 \leq j \leq r$, be diagonal matrices such that $F_{j}=A_{j}+D_{j}, 1 \leq j \leq r$, are nonsingular. If the matrices $2\left(A_{j}+D_{j}\right)-A, 1 \leq j \leq r$, are positive definite, then the splittings $A=F_{j}-G_{j}, 1 \leq j \leq r$, are $P$-regular, and so we can apply the above convergence results. 
On the other hand, the proof of Theorem 3.4 shows that $\left\|\left(F_{j}^{-1} G_{j}\right)\right\|_{\infty}<1,1 \leq$ $j \leq r$, is a sufficient condition for the convergence of Algorithm 2 for any matrix $A$, without additional hypotheses on the weighting matrices. So we have the following result.

Corollary 3.5. Let $A=F_{j}-G_{j}, 1 \leq j \leq r$, be splittings of the matrix $A \in \mathbb{C}^{n \times n}$ such that $\left\|\left(F_{j}^{-1} G_{j}\right)\right\|_{\infty}<1,1 \leq j \leq r$. If $q(l, j) \geq 1,1 \leq j \leq r, l=0,1,2, \ldots$, then the Non-stationary Multisplitting Algorithm 2 converges to the solution of the linear system (1.1), for any initial vector $x^{(0)}$.

Proof. Since $\left\|\left(F_{j}^{-1} G_{j}\right)\right\|_{\infty}<1,1 \leq j \leq r$, there exists a real constant $0 \leq \theta<1$ such that $\left\|\left(F_{j}^{-1} G_{j}\right)\right\|_{\infty} \leq \theta<1,1 \leq j \leq r$. By computing the infinite norm of $T^{(l)}$, defined in (3.3), we obtain for $l=0,1,2, \ldots,\left\|T^{(l)}\right\|_{\infty} \leq \max _{1 \leq j \leq r}\left\|F_{j}^{-1} G_{j}\right\|_{\infty}^{q(l, j)} \leq \theta<$ 1.

We note that Corollary 3.5 can be proved in the same way if the infinite norm is replaced with any matrix norm such that if for arbitrary matrices $T, T_{j}$ and weighting matrices $E_{j}, 1 \leq j \leq r$, such that $T=\sum_{j=1}^{r} E_{j} T_{j}$, then $\|T\| \leq \max _{1 \leq j \leq r}\left\|T_{j}\right\|$; see e.g., [4]. In particular one can use any weighted max-norm associated with a positive vector; see e.g., [15], [16] or [26] for descriptions and applications of these norms.

To finish this section we analyze the convergence of the Relaxed Non-stationary Multisplitting Algorithm 3. If in addition, we assume $0<\omega<\frac{2}{1+\max _{1 \leq j \leq r}\left\|F_{j}^{-1} G_{j}\right\|_{A}}$ in Theorem 3.3 and $0<\omega<\frac{2}{1+\max _{1 \leq j \leq r}\left\|F_{j}^{-1} G_{j}\right\|_{\infty}}$ in Corollary 3.5 the same results hold for Algorithm 3. Since its iteration matrices can be written as $\omega T^{(l)}+(1-\omega) I$, with $T^{(l)}$ defined in (3.3), the proofs follow in a similar way. Furthermore, if in Theorem 3.4 we assume $0<\omega<\frac{2}{1+\epsilon}$, then the convergence of Algorithm 3 also holds. So, the convergence of Algorithm 3 is shown under the same hypotheses as in the above results, setting $\omega$ in an interval $\left(0, \omega_{0}\right)$, with $\omega_{0}>1$.

\section{Asynchronous iterations}

The motivation of defining an asynchronous non-stationary method is to obtain a parallel implementation of the non-stationary iterative methods on a multiprocessor system such that the communication and synchronization between the cooperating processes are reduced. To illustrate that, consider Algorithm 2; this algorithm is synchronous in the sense that step (1.6) is performed only after all processors have computed their iterate vectors $y_{j}^{(q(l, j))}, 1 \leq j \leq r$. Alternatively, we can consider a parallel implementation of a non-stationary multisplitting method in which each part of $x^{(l+1)}$, i.e., $E_{j} y_{j}^{(q(l, j))}$, can be updated without waiting for the other parts of $x^{(l+1)}$ to be updated. In order to construct an asynchronous version of Algorithm 2, we consider a different scheme where all processors are always working without waiting for information from the other processors. In a formal way, let $\left\{j_{l}\right\}_{l=0}^{\infty}, 1 \leq j_{l} \leq r$, be a sequence of integers that indicates the processor which updates the approximation to the solution at the $l$ th iteration. Let $r_{l}-1$ be the number of times that processors other than the $j_{l}$ th processor update the approximation of the solution during the time interval in which the $j_{l}$ th processor's 
calculations are performed. This implies that $r_{l}$ is the smallest positive integer such that $j_{l}=j_{l+r_{l}}$. Assume that there exists a positive integer $K$ such that $0 \leq r_{l}-1<K$, that is, in carrying out the evaluation of the $l$ th iterate, a process cannot make use of any value of the components of the $j$ th iterate if $j<l-K$. With this notation we consider the following asynchronous scheme

$$
x^{\left(l+r_{l}\right)}=\left(I-E_{j_{l}}\right) x^{\left(l+r_{l}-1\right)}+E_{j_{l}} P_{j_{l}}^{q\left(l, j_{l}\right)} x^{(l)}, \quad l=0,1,2, \ldots,
$$

where the operators $P_{j}, 1 \leq j \leq r$, are defined in (1.4) and $x^{(-K)}=x^{(-K+1)}=$ $\cdots=x^{(0)}$. As it can be appreciated, scheme (4.1) corresponds to the following algorithm.

Algorithm 4. (Asynchronous Non-Stationary Multisplitting).

Given the initial vectors $x^{(-K)}=x^{(-K+1)}=\cdots=x^{(0)}$.

In processor $j_{l}, l=0,1,2, \ldots$, until convergence.

$$
\begin{gathered}
y_{j_{l}}^{(0)}=x^{(l)} \\
\text { For } k=1 \text { to } q\left(l, j_{l}\right) \\
F_{j_{l}} y_{j_{l}}^{(k)}=G_{j_{l}} y_{j_{l}}^{(k-1)}+b \\
x^{\left(l+r_{l}\right)}=\left(I-E_{j_{l}}\right) x^{\left(l+r_{l}-1\right)}+E_{j_{l}} y_{j_{l}}^{\left(q\left(l, j_{l}\right)\right)} .
\end{gathered}
$$

In the same way as in the synchronous case, a relaxation parameter $\omega \in \mathbb{R}, \omega \neq 0$, can be introduced in Algorithm 4, by replacing the vector $y_{j_{l}}^{\left(q\left(l, j_{l}\right)\right)}$ in (4.2) with the equation $\omega y_{j_{l}}^{\left(q\left(l, j_{l}\right)\right)}+(1-\omega) x^{(l)}$. When $\omega \neq 1$ we have a Relaxed Asynchronous Non-stationary Multisplitting Algorithm (Algorithm 5).

The following theorem shows the convergence of Algorithm 4 under similar hypotheses as those for the synchronous Algorithm 2 in Theorem 3.3.

Theorem 4.1. Let $A$ be a hermitian positive definite matrix. Let $A=F_{j}-$ $G_{j}, 1 \leq j \leq r$, be $P$-regular splittings and $E_{j}=\alpha_{j} I, 1 \leq j \leq r$, with $0<\alpha_{j} \leq 1$. Assume that there exists a positive integer $K$ such that $0 \leq r_{l}-1<K$. If $q(l, j) \geq 1,1 \leq j \leq r, l=0,1,2, \ldots$, then the Asynchronous Non-stationary Multisplitting Algorithm 4 converges to the solution of the linear system (1.1), for any initial vector $x^{(0)}$.

Proof. In order to analyze the convergence of the asynchronous iteration (4.1) (or Algorithm 4), we will construct a procedure in $\mathbb{C}^{n K}$, with $K$ satisfying $0 \leq r_{l}-1<$ $K$. To this purpose, let us use the following notation. Let $\xi$ be the unique solution of the linear system (1.1) and let $e^{(l)}=x^{(l)}-\xi$ be the error vector in the $l$ th iteration of the asynchronous scheme (4.1). Define

$$
\bar{e}_{l}=\left(\left(e^{(l)}\right)^{T},\left(e^{(l-1)}\right)^{T}, \ldots,\left(e^{(l-K+1)}\right)^{T}\right)^{T} \in \mathbb{C}^{n K} .
$$

Since $0 \leq r_{l}-1<K$, it follows that $l+r_{l}-K \leq l \leq l+r_{l}-1, l=0,1,2, \ldots$, and then we can write

$$
e^{(l)}=S_{l} \bar{e}_{l+r_{l}-1},
$$

where $S_{l}, l=0,1,2, \ldots$, is an $n \times n K$ matrix with an $n \times n$ identity block in the $r_{l}$ position and the remaining $K-1$ blocks are zero. From (4.1) and (4.4), and knowing that $E_{j}=\alpha_{j} I, 1 \leq j \leq r$, it follows that

$$
\bar{e}_{l+r_{l}}=B_{l+r_{l}} \bar{e}_{l+r_{l}-1},
$$


where $B_{l+r_{l}} \in \mathbb{C}^{n K \times n K}$ is defined as

$$
B_{l+r_{l}}=\left[\begin{array}{ccccc}
\left(1-\alpha_{j_{l}}\right) I & O & \ldots & O & O \\
I & O & \ldots & O & O \\
\vdots & \vdots & & \vdots & \vdots \\
O & O & \ldots & I & O
\end{array}\right]+\left[\begin{array}{c}
\alpha_{j_{l}}\left(F_{j_{l}}^{-1} G_{j_{l}}\right)^{q\left(l, j_{l}\right)} S_{l} \\
O \\
\vdots \\
O
\end{array}\right] .
$$

Then $\bar{e}_{l+K}=B_{l+K} B_{l+K-1} \cdots B_{l+1} \bar{e}_{l}$.

Let $\mu$ be an arbitrary nonzero $n K$-dimensional vector partitioned as in (4.3), that is, $\mu=\left(\mu^{i}\right)_{i=1}^{K} \in \mathbb{C}^{n K}$, with $\mu^{i} \in \mathbb{C}^{n}$. We define a vector norm on $\mathbb{C}^{n K}$ by

$$
\|\mu\|=\max _{1 \leq i \leq K}\left\|\mu^{i}\right\|_{A}
$$

and its induced matrix norm on $\mathbb{C}^{n K \times n K}$. We show that there exists a real constant $0 \leq \gamma<1$, such that $\left\|B_{l+K} B_{l+K-1} \cdots B_{l+1}\right\| \leq \gamma<1, \quad l=0,1,2, \ldots$, and therefore $\lim \bar{e}_{l}=0$.

Since $\stackrel{l \rightarrow \infty}{=} F_{j}-G_{j}, 1 \leq j \leq r$, are $P$-regular splittings, reasoning as in Theorem 3.3 it follows that there exists a real constant $0 \leq \theta<1$ such that $\left\|F_{j}^{-1} G_{j}\right\|_{A} \leq \theta$, and then $\left\|\left(F_{j}^{-1} G_{j}\right)^{s}\right\|_{A} \leq \theta<1$, for all $s \geq 1,1 \leq j \leq r$. Let

$$
v_{\nu}=B_{l+\nu} B_{l+\nu-1} \cdots B_{l+1} \mu:=\left[\begin{array}{c}
v_{\nu}^{1} \\
v_{\nu}^{2} \\
\vdots \\
v_{\nu}^{K}
\end{array}\right], \quad v_{\nu}^{i} \in \mathbb{C}^{n}, i=1,2, \ldots, K, \nu \geq 1 .
$$

From (4.4) and (4.5), $v_{1}^{1}=\left(1-\alpha_{j_{l}}\right) \mu_{1}+\alpha_{j_{l}}\left(F_{j_{l}}^{-1} G_{j_{l}}\right)^{q\left(l, j_{l}\right)} \mu_{i}, 1 \leq i \leq K$. Then

$$
\begin{aligned}
\left\|v_{1}^{1}\right\|_{A} & \leq\left(1-\alpha_{j_{l}}\right)\left\|\mu_{1}\right\|_{A}+\alpha_{j_{l}}\left\|\left(F_{j_{l}}^{-1} G_{j_{l}}\right)^{q\left(l, j_{l}\right)}\right\|_{A}\left\|\mu_{i}\right\|_{A} \\
& \leq\left[\left(1-\alpha_{j_{l}}\right)+\alpha_{j_{l}} \theta\right]\|\mu\| \leq \gamma\|\mu\|,
\end{aligned}
$$

where $\gamma=\max _{1 \leq j \leq r}\left[\left(1-\alpha_{j}\right)+\alpha_{j} \theta\right]<1$. Then, from (4.5)

$$
\left\|v_{1}^{i}\right\|_{A}=\left\|\mu^{i-1}\right\|_{A} \leq\|\mu\|, \quad i=2,3, \ldots, K .
$$

Now, from expression (4.6), $v_{\nu}^{1+i}=v_{\nu-1}^{i}, i=1,2, \ldots, K-1$. Then, using the bounds (4.7) and (4.8) it follows that

$$
\left\|v_{\nu}^{i}\right\|_{A} \leq\left\{\begin{aligned}
\gamma\|\mu\|, & i=1,2, \ldots, \nu \\
\|\mu\|, & i=\nu+1, \ldots, K
\end{aligned}\right.
$$

Hence, $\left\|v_{K}\right\| \leq \gamma\|\mu\|$, for all nonzero vectors $\mu \in \mathbb{C}^{n K}$. Therefore, we have that $\left\|B_{l+K} B_{l+K-1} \cdots B_{l+1}\right\| \leq \gamma<1, \quad l=0,1,2, \ldots$. Thus, the proof is complete.

From now on, we assume, as is customary in the description of asynchronous algorithms (see e.g., [3] and [9]), that the sequence of integers $\left\{j_{l}\right\}_{l=0}^{\infty}, 1 \leq j_{l} \leq r$, is a regulated sequence. This means that there exists a positive integer $K$, such that each of the integers $1,2, \ldots, r$, appears at least once in every $K$ consecutive elements of the sequence. That actually implies $0 \leq r_{l}-1<K$. We point out that in Theorem 4.1 we have only needed the latter bounds; this is due to the fact that the weighting matrices are of the form $E_{j}=\alpha_{j} I, 1 \leq j \leq r$. Another similar way of describing asynchronous iterations has been considered by Frommer [10] and other authors, e.g., Bru, Migallón, Penadés and Szyld [7] and the authors cited therein. 
Now, we study the convergence of the asynchronous scheme (4.1) (or Algorithm 4) under hypotheses similar to those of Theorem 3.4 and Corollary 3.5.

Theorem 4.2. Let $A$ be a hermitian positive definite matrix. Let $A=F_{j}-G_{j}, 1 \leq$ $j \leq r$, be $P$-regular splittings. Assume further that $\lim _{l \rightarrow \infty} q(l, j)=\infty, 1 \leq j \leq r$. Given a regulated sequence of integers $\left\{j_{l}\right\}_{l=0}^{\infty}, 1 \leq j_{l} \leq r$, the Asynchronous Nonstationary Multisplitting Algorithm 4 converges to the solution of the linear system (1.1), for any initial vector $x^{(0)}$.

Proof. Reasoning as in Theorem 3.4 there exists an $l_{0}$ and a real constant $\theta$, such that $\left\|\left(F_{j}^{-1} G_{j}\right)^{q(l, j)}\right\|_{\infty} \leq \theta<1$, for all $l \geq l_{0}$. Thus, setting $z=(1,1, \ldots, 1)^{T}$,

$$
\left|\left(F_{j}^{-1} G_{j}\right)^{q(l, j)}\right| z \leq \theta z, \quad l \geq l_{0} .
$$

By using error analysis as in Theorem 4.1 and reasoning in a similar way as in the proof of [20, Theorem 3.2], which in turn is based on [3, Theorem 2.2], it follows that

$$
\left|B_{l+2 K-1} B_{l+2 K-2} \cdots B_{l+1}\right| z \leq \theta z, \quad l \geq l_{0},
$$

where the matrices $B_{l}$ are obtained by replacing $\left(1-\alpha_{j_{l}}\right) I$ in (4.5) with $I-E_{j_{l}}$. Then,

$$
\left\|B_{l+2 K-1} B_{l+2 K-2} \cdots B_{l+1}\right\|_{\infty} \leq \theta<1, \quad l \geq l_{0} .
$$

Thus, the proof is completed.

Corollary 4.3. Let $A=F_{j}-G_{j}, 1 \leq j \leq r$, be splittings of the matrix $A \in$ $\mathbb{C}^{n \times n}$ such that $\left\|\left(F_{j}^{-1} G_{j}\right)\right\|_{\infty}<1,1 \leq j \leq r$. Assume $q(l, j) \geq 1,1 \leq j \leq$ $r, l=0,1,2, \ldots$ Given a regulated sequence of integers $\left\{j_{l}\right\}_{l=0}^{\infty}, 1 \leq j_{l} \leq r$, the Asynchronous Non-stationary Multisplitting Algorithm 4 converges to the solution of the linear system (1.1), for any initial vector $x^{(0)}$.

Proof. The proof follows in a similar way as in the proof of Theorem 4.2.

Analogously, setting $\omega$ as at the end of Section 3, the convergence of Algorithm 5 is done under the same hypotheses as in the above results.

\section{REFERENCES}

[1] Michele Benzi and Daniel B. Szyld, Existence and uniqueness of splittings for stationary iterative methods with applications to alternating methods, Tech. Report 95-81, Department of Mathematics, Temple University, Philadelphia, Pa., August 1995. Also available via anonymous ftp at ftp.math.temple.edu in directory pub/szyld. To appear in Numer. Math.

[2] Abraham Berman and Robert J. Plemmons, Nonnegative matrices in the mathematical sciences, third ed., Academic Press, New York, 1979, Reprinted by SIAM, Philadelphia, 1994. MR 95e: 15013

[3] Rafael Bru, Ludwing Elsner, and Michael Neumann, Models of parallel chaotic iteration methods, Linear Algebra Appl. 103 (1988), 175-192. MR 90b:65255

[4] Rafael Bru and Robert Fuster, Parallel chaotic extrapolated Jacobi method, Appl. Math. Lett. 3 (1990), no. 4, 65-69. CMP 91:04

[5] Rafael Bru, Violeta Migallón, and José Penadés, Chaotic inner-outer iterative schemes, Proceedings of the Fifth SIAM Conference on Applied Linear Algebra (J.G. Lewis, ed.), SIAM Press, Philadelphia, 1994, pp. 434-438.

[6] _ Chaotic methods for the parallel solution of linear systems, Computing Systems in Engineering 6 (1995), no. 4,5, 385-390.

[7] Rafael Bru, Violeta Migallón, José Penadés, and Daniel B. Szyld, Parallel, synchronous and asynchronous two-stage multisplitting methods, Electronic Transactions on Numerical Analysis 3 (1995), 24-38. MR 95m:65048 
[8] D. Chazan and W. Miranker, Chaotic relaxation, Linear Algebra Appl. 2 (1969), 199-222. MR 40:5114

[9] Ludwig Elsner, Israel Koltracht, and Michael Neumann, On the convergence of asynchronous paracontractions with application to tomographic reconstruction from incomplete data, Linear Algebra Appl. 130 (1990), 65-82. MR 91g:65093

[10] Andreas Frommer, On asynchronous iterations in partially ordered spaces, Numer. Funct. Anal. Optim. 12 (1991), no. 3,4, 315-325. MR 92m:54060

[11] Andreas Frommer and Günter Mayer, Convergence of relaxed parallel multisplitting methods, Linear Algebra Appl. 119 (1989), 141-152. MR 90f:65049

[12] _ On the theory and practice of multisplitting methods in parallel computation, Computing 49 (1992), 63-74. MR 93e:65158

[13] Andreas Frommer and Daniel B. Szyld, Asynchronous two-stage iterative methods, Numer. Math. 69 (1994), 141-153. MR 95m:65049

[14] Robert Fuster, Violeta Migallón, and José Penadés, Non-stationary parallel multisplitting AOR methods, Electronic Transactions on Numerical Analysis 4 (1996), 1-13. MR 96m:65038

[15] _ Parallel chaotic extrapolated Jacobi-like methods, Linear Algebra Appl. 247 (1966), 237-250. CMP 97:02

[16] Alston S. Householder, The theory of matrices in numerical analysis, Blaisdell, Waltham, Mass. 1964, Reprinted by Dover, New York, 1975. MR 51:14539

[17] Charles R. Johnson and Rafael Bru, The spectral radius of a product of nonnegative matrices, Linear Algebra Appl. 141 (1990), 227-240. MR 91i:15011

[18] Paul J. Lanzkron, Donald J. Rose, and Daniel B. Szyld, Convergence of nested iterative methods for linear systems, Numer. Math. 58 (1991), 685-702. MR 92e:65045

[19] Guri I. Marchuk, Splitting and alternating direction methods, Handbook of Numerical Analysis, Vol. I (P.G. Ciarlet and J.L. Lions, eds.), North Holland, New York, 1990, pp. 197-462. CMP 90:08

[20] José Mas, Violeta Migallón, José Penadés, and Daniel B. Szyld, Non-stationary parallel relaxed multisplitting methods, Linear Algebra Appl. 241-243 (1996), 733-748. CMP 96:15

[21] Reinhard Nabben, A note on comparison theorems for splittings and multisplittings of hermitian positive definite matrices, Linear Algebra Appl. 233 (1995), 67-80. MR 97a:15035

[22] Michael Neumann and Robert J. Plemmons, Convergence of parallel multisplitting iterative methods for M-matrices, Linear Algebra Appl. 88-89 (1987), 559-573. MR 88k:65143

[23] Dianne P. O'Leary and Robert E. White, Multi-splittings of matrices and parallel solution of linear systems, SIAM J. Alg. Disc. Meth. 6 (1985), 630-640. MR 86h:65047

[24] James M. Ortega, Matrix theory, Plenum Press, New York, 1987. MR 88a:15002

[25] James M. Ortega, Introduction to parallel and vector solution of linear systems, Plenum Press, New York, 1988. MR 92i:65220a

[26] Werner C. Rheinboldt and James S. Vandergraft, A simple approach to the Perron-Frobenius theory for positive operators on general partially-ordered finite-dimentional linear spaces, Math. Comp. 27 (1973), 139-145. MR 48:3997

[27] F. Robert, M. Charnay, and F. Musy, Itérations chaotiques série-parallèle pour des équations non-linéaires de point fixe, Aplikace Matematiky 20 (1975), 1-38. MR 51:9472

[28] Robert E. White, Multisplittings and parallel iterative methods, Comput. Methods Appl. Mech. Engrg. 64 (1987), 567-577. MR 88j:65315

[29] _ Multisplitting with different weighting schemes, SIAM J. Matrix Anal. Appl. 10 (1989), 481-493. MR 90k:65116

[30] Multisplitting of a symmetric positive definite matrix, SIAM J. Matrix Anal. Appl. 11 (1990), 69-82. MR 91k:65064

Departamento de Tecnología Informática y Computación, Universidad de Alicante, E-03071 Alicante, Spain

E-mail address: chus@dtic.ua.es

E-mail address: violeta@dtic.ua.es

E-mail address: jpenades@dtic.ua.es 